\title{
International Journal \\ of Cancer and Oncology
}

\section{A Patient with Testicular Seminoma, Inguinal Lymphadenopathy and Abnormal PET Scan}

\section{Sonya Minmin Chew ${ }^{1}$, Geoffrey Alan Watson ${ }^{1}$, Sarah Picardo ${ }^{1}$, Vourneen Healy ${ }^{2}$, Padraig O'Brien ${ }^{3}$, Rajnish Kumar Gupta ${ }^{1,4^{*}}$}

${ }^{1}$ Department of Medical Oncology, University Hospital Limerick, Ireland

${ }^{2}$ Department of Histopathology, University Hospital Limerick, Ireland

${ }^{3}$ Department of Radiology, University Hospital Limerick, Ireland

${ }^{4}$ Graduate Entry Medical School, University Hospital Limerick, Ireland

*Corresponding author: Rajnish Kumar Gupta, Department of Medical Oncology, Mid-Western Cancer Centre, University Hospital Limerick, Limerick, Ireland, Tel: +35361482542; E-mail: rajnish.gupta@hse.ie

\section{Abstract}

Testicular malignancies are rare but are associated with an excellent prognosis. The majority of patients are diagnosed at an early stage, and even in the presence of nodal or distant metastases, treatment is administered with curative intent. Embryologically the male genital system is derived from the dorsal abdominal wall at level of the lumbar vertebrae, and migrates caudally towards the deep inguinal rings during development. Thus nodal metastases most often occur along the para-aortic lymphatic pathway up to this lumbar level. Inguinal metastases in testicular cancer are exceedingly rare, and often suggest disruption of this lymphatic pathway. We report the case of a young gentleman diagnosed with good risk testicular seminoma with inguinal metastases with a background of previous scrotal surgery and vasectomy.
Received Date: January 12, 2017

Accepted Date: February 8, 2017

Published Date: February 13, 2017

Citation: Gupta, R.K., et al. A Patient with Testicular Seminoma, Inguinal Lymphadenopathy and Abnormal PET Scan. (2017) Int J Cancer Oncol 4(2): 199- 202.

DOI: $10.15436 / 2377-0902.17 .1310$

\section{Case Report}

A 43 year old gentleman presented with a three week history of painless right testicular swelling. He denied any systemic symptoms and was otherwise well. Testicular ultrasound revealed a solid right epididymal mass, concerning for malignancy. Tumour markers (including human chorionic gonadotrophin, alpha fetoprotein and lactate dehydrogenase) were normal. A staging CT scan revealed pathological right external iliac and right inguinal lymphadenopathy. A positron emission tomography (PET) scan showed a flurodeoxyglucose (FDG)-avid right external iliac lymph node, two FDG-avid lymph nodes in the inguinal region, the largest measuring $2.1 \mathrm{~cm}$, and an FDG-avid left inguinal lymph node. There were no other pelvic nodes seen.

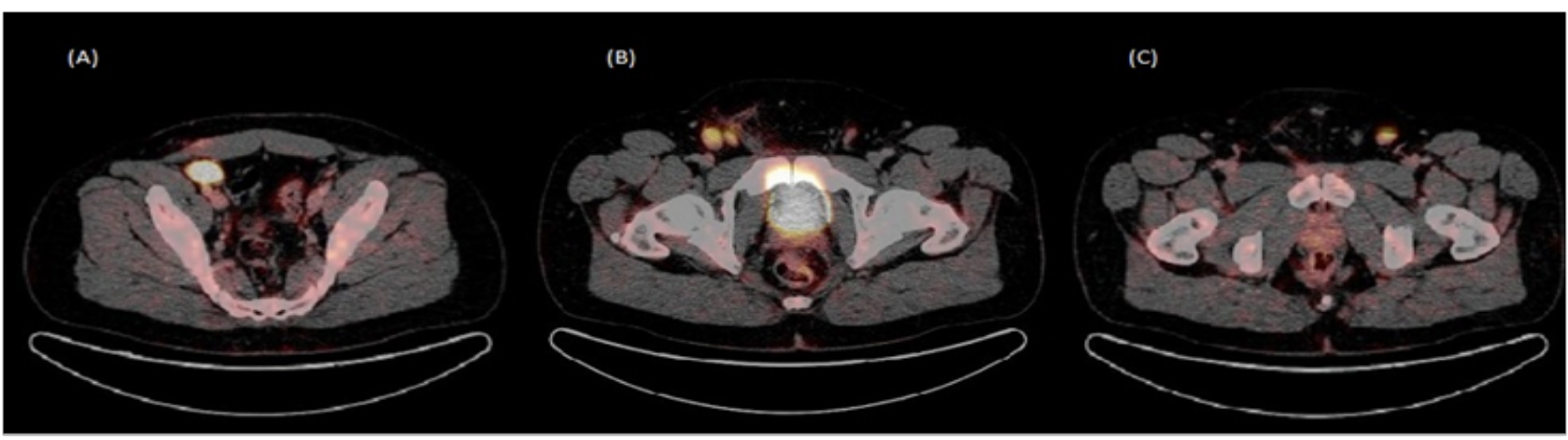

Figure 1: - Post operative staging PET scan.

(A) $3 \mathrm{~cm}$ FDG-avid right external iliac lymph node

(B) Two FDG-avid lymph nodes in the right inguinal region, the largest measuring at $2.1 \mathrm{~cm}$

(C) $1.8 \mathrm{~cm}$ FDG-avid left inguinal lymph node

Copyrights: (C) 2017 Gupta, R.K. This is an Open access article distributed under the terms of Creative Commons Attribution 4.0 International License. 
His background was significant for a right orchidopexy for an undescended testis performed 32 years previously. He also underwent a vasectomy 5 years ago which included the removal of a cyst from the right epididymis through a scrotal incision. He had no history of mumps. He was an ex-smoker and drank alcohol in moderation. There was no family history of cancer. He was not on any regular medications and had no drug allergies.

He underwent a right radical orchidectomy without complications. Pathological examination of the test is revealed a seminoma with lymphovascular invasion and clear resected margins and was staged as $\mathrm{pT}_{2}$. In addition, the patient underwent a right groin lymph node biopsy which confirmed a metastatic seminoma and therefore his final clinical and pathological stage was $\mathrm{pT}_{2} \mathrm{pN}_{2} \mathrm{M}_{0} \mathrm{~S}_{0}$ stage IIB. He proceeded to have three cycles of BEP (bleomycin, etoposide, cisplatin) chemotherapy which he tolerated well. Restaging scans post treatment revealed a complete radiological response. He remains well and in remission on follow up.

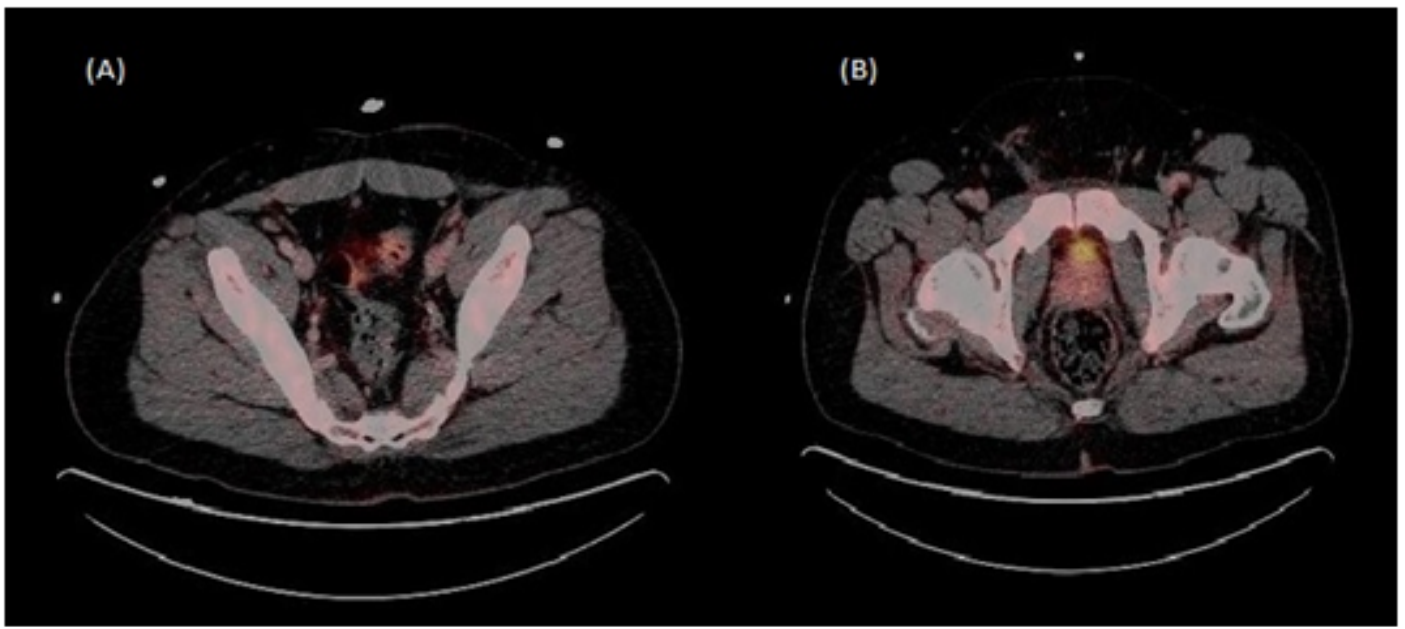

Figure 2: PET scan post chemotherapy.

(A) and (B) Complete resolution of external iliac and inguinal lymph nodes. No abnormal tracer uptake.

\section{Discussion}

Testicular cancer represents one of the true success stories in modern oncology. The discovery of a successful three drug regimen (BEP) to treat these malignancies has resulted in excellent cure rates with 5 year relative overall survival (OS) of $96 \%{ }^{[1]}$. OS rates in seminomas are $99 \%$, which may reflect the fact that most cases are diagnosed early with stage 1 disease ${ }^{[1]}$. Thus the focus today has shifted towards reducing the 'burden of cure', namely the long term complications of systemic chemotherapy.

Testicular cancer is relatively rare, accounting for $1-2 \%$ of male neoplasm $\mathrm{s}^{[1]}$. However in young patients, it is one of the most common cancers, representing $30 \%$ of all cancers in 25 - 39 year olds ${ }^{[1]}$. Pathological classification is divided into predominantly germ cell tumours (90 - 95\%of cases), sex cord tumours and miscellaneous non-specific stromal tumours ${ }^{[2]}$. There are three broad entities of germ cell-derived tumours in the human testis: the infantile teratomas or yolk sac tumours, the seminomas and nonseminomas, and the spermatocytic seminomas. The most recent data from Ireland revealed 57\% of these are seminomatous and $40 \%$ non-seminomatous. Non germ-cell subtypes, of which less than 2 percent are diagnosed per year, include sarcomas and sex cord tumours ${ }^{[1,3]}$. These sub-types may also vary with age. In Ireland non-seminomatous tumours appear to be most common in younger patients and comprise up to $80 \%$ of testicular tumours in patients under 20 years of age ${ }^{[1]}$. However over $80 \%$ of tumours diagnosed in patients aged 40 or over were seminomatous cell type $\mathrm{e}^{[1]}$.

During embryological development, the testes migrate from their early developmental lumbar position, in the dorsal abdominal wall, to the deep inguinal rings above the scrotum. This occurs between the $7^{\text {th }}$ week of gestation to the end of 40 weeks at full term. The descent of the testis descent is thought to have two phases. The first is trans abdominal descent, and is dependent on insulin-like hormone 3 (INSL3). The second is inguinoscrotal descent, dependent on androgens. Anomalies of this migration include cryptorchidism, where the testis may be found in the pelvic, iliac, or even lumbar regions, and represents a major risk factor for the development of testicular malignan$\mathrm{cy}^{[4]}$, as was the case with this patient.

The testicular lymphatics typically drain along the gonadal vessels, ascending through the spermatic cord, continuing past the psoas muscle and reaching the retroperitoneal nodes via the para-aortic lymphatic pathway, located between the lower thoracic and lumbar vertebrae. Thus the retroperitoneal lymph nodes are frequently observed as a site of metastasis ${ }^{[5]}$. From these nodes, metastatic disease may spread downward in a retrograde fashion, towards the aortic bifurcation. Rarely surgery, chemotherapy, and radiation therapy may disrupt this lymphatic pathway and modify the pattern of nodal disease ${ }^{[6]}$.

The superficial inguinal lymph nodes typically drain the regions of the skin from the lower abdomen, part of the buttocks and scrotum, the perineum and the penis, which in turn drain into the deep inguinal nodes, found under the fascia lata. However, traumatic disruption of scrotal lymphatics result in the anastomosis of collateral vessels to a new drainage system, resulting in an alternative route of spread to inguinal nodes. Types of surgery that can facilitate this spread include previous testicular surgery and manipulation such as orchidopexy, trans-scrotal biopsy, varicocelectomy and hydrocoele repair ${ }^{[7]}$. Tumour extension into the epididymis, vas deferens or scrotal wall may also 
be a rare cause ${ }^{[8]}$. The patient described in this report had two scrotal surgical procedures carried out prior to his diagnosis, resulting in probable disruption of lymphatic drainage from his right testicle.

As previously mentioned inguinal lymph node metastases from a testicular primary is rare. In one large study by Daugaard et al., which included 695 patients diagnosed with Stage 1 testicular cancer, inguinal lymph node involvement was observed in only $2 \%$ of these patients ${ }^{[9]}$. The authors reported the incidence to be more common in non-seminomatous germ cell tumours $(4 \%)$ compared to seminomas $(0.5 \%)^{[9]}$. Interestingly, in our patient, a CT scan demonstrated unilateral inguinal lymph node enlargement by radiological criteria, while PET confirmed additional FDG avidity in the contralateral inguinal region. Some studies have suggested that PET may be superior to conventional CT imaging for staging of testicular cancer, with highest sensitivity in seminoma ${ }^{[10,11]}$. PET is particularly useful in the case of lymph nodes that are not pathologically enlarged on CT but with clinical or biochemical suspicion of disease ${ }^{[10]}$.

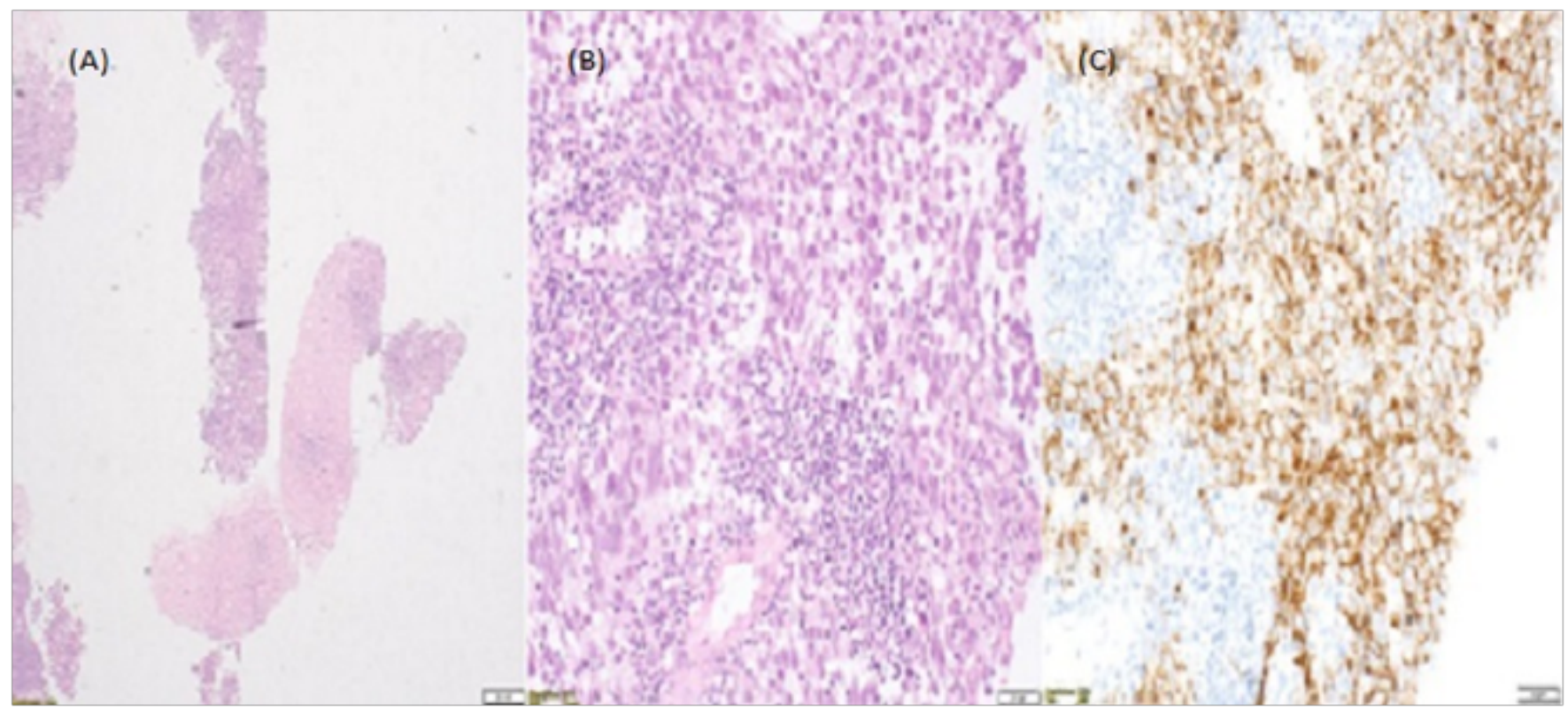

Figure 3:

(A) Right inguinal lymph node core biopsies

(B) Seminoma haematoxylin-eosin stain

(C)Seminoma C-Kit stain

Orchidectomy followed by surveillance commonly provides cure in patients with stage 1 testicular germ cell tumours. The AJCC classification stratifies patients using both TNM staging and tumour marker levels in order to assess the need for chemotherapy. In the case of our patient, staged as stage $\mathrm{IIb}$, chemotherapy in the form of BEP was recommended. Management of the inguinal lymphatics in patients with testicular tumours and a previous history of inguinal or scrotal surgery remains controversial, largely due to insufficient data ${ }^{[11]}$. Surveillance may be augmented with chemotherapy with either carboplatin or the BEP regime ${ }^{[12]}$. Prophylactic inguinal lymph adenectomy is rarely mentioned, while in some reports patients have been found to have positive inguinal nodes with no retroperitoneal lymphadenopathy ${ }^{[13,14]}$. Thus it has been postulated that ipsilateral inguinal and bilateral retroperitoneal node dissection may be considered as part of the primary therapy for non-seminomatous testicular tumours with a previous history of scrotal and inguinal procedures ${ }^{[13]}$. However, Mianne et al, also suggested that prophylactic ipsilateral inguinal dissection is not necessary in patients with non-seminomatous testicular tumors with a history of inguinal or scrotal surgery, owing to the efficacy of primary and secondary chemotherapy ${ }^{[15]}$. Our patient had bilateral inguinal lymph node involvement at diagnosis and had a complete response to chemotherapy, suggesting that inguinal dissection may have resulted in significant morbidity without conferring any obvious benefit.

\section{Conclusion}

Management of testicular cancer depends on accurate staging of the disease, and almost all patients should be managed with curative intent. Surgical excision remains the primary treatment modality, with chemotherapy, and in certain cases radiotherapy recommended in advanced disease. Due to embryological development the retroperitoneal lymph nodes are the most common site of metastasis, while disruption of normal lymphatic drainage due to previous scrotal surgery should raise suspicion of a less frequent site of lymphatic involvement. Staging of a patient with suspicion of inguinal lymph node involvement needs to include PET imaging for full assessment. 


\section{References}

1. Cancer in Ireland. (2011) Annual report of the National Cancer Registry, NCRI

2. Albers, P., Albrecht, W., Algaba, F., et al. Guidelines on testicular cancer: 2015 update. (2015) Eur Urol 68 (2015): 1054-1068.

3. Looijenga, L.H., Oosterhuis, J.W. Pathogenesis of testicular germ cell tumors. (1999) Rev Reprod 4(2): 90-100.

4. Pansky, B. Review of Medical Embryology Chapter 103. Inguinal Canal Development and Testicular Migration. (1982) California: Embryome Sciences.

5. Jamieson, J.K., Dobson, J.F. The lymphatics of the testicle. (1910) Lancet 1: 493-495.

6. Paño, B., Sebastià, C., Buñesch, L., et al. Pathways of lymphatic spread in male urogenital pelvic malignancies. (2011) Radio Graphics 31(1): 135-160.

7. Ismail, M., Zaman, F., Baithun, S., et al. Inguinal lymph node metastases from a testicular seminoma: a case report and a review of the literature. (2010) J Med Case Rep 4: 378

8. Shah, S., Bakshi, H. Inguinal lymphnode metastatic testicular seminoma: A case report and review of literature. (2012) Indian J Surg Oncol 3(1): 38-40.

9. Daugaard, G., Karas, V., Sommer, P. Inguinal metastases from testicular cancer. (2006) BJU Int 97(4): 724-726.

10. Lassen, U., Daugaard, G., Eigtved, A., et al. Whole-body FDG-PET in patients with stage I non-seminomatous germ cell tumours. (2003) Eur J Nucl Med Mol Imaging 30(3): 396-402.

11. Ambrosini, V., Zucchini, G., Nicolini, S., et al. 18F-FDG PET/CT impact on testicular tumours clinical management. (2014) Eur J Nucl Med Mol Imaging 41(4): 668-673.

12. Bantis, A., Sountoulides, P., Kalaitzis, C., et al. Single inguinal node metastasis of stage I testicular seminoma shown by scrotal scintigraphy, 6 months following radical orchiectomy. (2012) Hell J Nucl Med 15(2): $150-152$.

13. Wheeler, J.S. Jr, Babayan, R.K., Hong, W.K., et al. Inguinal node metastases from testicular tumors in patients with prior orchiopexy. (1983) J Urol 129(6): 1245-1247.

14. Johnson, D.E., Babaian, R.J. The case for conservative surgical management of the ilioinguinal region after inadequate orchiectomy. (1980) J Urol 123(1): 44-46.

15. Mianné, D.M., Barnaud, P., Altobelli, A., et al. Inguinal lymphatic metastasis of cancer of the testis: staging and therapeutic approach. (1991) Ann Urol (Paris) 25(4): 199-202. 